\title{
Shock-induced two-phase flows in an aligned baffle system filled with suspended particles
}

\author{
B.Y. Wang ${ }^{1}$, Y.H. $\mathrm{Xu}^{2}$, Q.S. $\mathrm{Wu}^{2}$ \\ ${ }^{1}$ Institute of Mechanics, Chinese Academy of Sciences, Beijing 100080, P.R. China \\ 2 Department of Modern Mechanics, University of Science and Technology of China, Hefei 230026, P.R. China
}

Received 23 October 1995 / Accepted 6 June 1996

\begin{abstract}
This paper describes the shock propagation through a dilute gas-particle suspension in an aligned baffle system. Numerical solution to two-phase flows induced by a planar shock wave is given based on the two-continuum model with interphase coupling. The governing equations are numerically solved by using high-resolution schemes. The computational results show the shock reflection and diffraction patterns, and the shock-induced flow fields in the 4-baffle system filled with the dusty gas.
\end{abstract}

Key words: Shock propagation, Two-phase flow, Baffle system, Numerical solution

\section{Introduction}

Shock-induced flows have been an avid interest for more than 100 years of research (Hillier 1993). Most of the existing work concerns mainly the shock phenomena in pure gases. Recently, relevant investigations are extended to treat the problem of shock reflection and diffraction in various gas-particle suspensions (i.e. dusty gases) since many applications (dust explosion, ablation and erosion, etc.) involve such a dusty gas. For instance, shock propagation in baffle systems filled with a dilute dusty gas and the post-shock flow in the two-phase medium are important for explosion safety analyses.

In this work, numerical simulation of the two-phase flow induced by a planar incident shock wave in an aligned twodimensional baffle system is performed. Some experimental and computational studies have been done for the pure-gas case (Reichenbach and Kuhl 1993; Luo et al. 1993) but none for the dusty-gas case until now. To formulate the unsteady compressible two-phase flow in these systems with complex geometry, two-dimensional and time-dependent Euler equations are presented based on the two-continuum model, where the interaction between the gas and particles is taken into account. The governing equations are solved by using the GRP scheme (Ben-Artzi and Falcovitz 1984) and NND scheme (Zhang and Li 1992), which are adopted respectively to the gas and particle phases. The dusty shock reflection and diffraction patterns and the unsteady two-phase flow structures behind the shock front are obtained for a twodimensional baffled tube. These computational results reveal the fundamental features of the post-shock flow field: turbulent mixing and particle relaxation consist of the dissipation mechanisms of the shock strength and speed.

\section{Basic assumptions and governing equations}

As mentioned above, the problem under consideration is the shock-induced flows due to the reflection and diffraction of a planar incident shock wave propagating in an aligned 2-D baffle system filled with a dilute dusty gas. For the analysis of this two-phase flow, the two-continuum model is employed and the following assumptions are included: (1) The gas phase is a compressible and perfect gas. Its viscosity and thermal conductivity are ignored except for the interaction between the gas and particles. (2) The particle phase consists of rigid and inert particles. They are uniform solid spheres without Brownian motion and internal temperature gradient. (3) The volume fraction of the particle phase is negligible and there is no mutual interaction among the particles. (4) In this dilute gas-particle system, the partial pressure due to particle contribution can be neglected. (5) There are momentum and energy (but no mass) exchanges between the gas and particles. Therefore, in addition to the interphase force, the effect of heat transfer on the two-phase flow is included. (6) The only force on the particles to be taken into account is viscous drag since the other mechanisms such as pressure gradient force, virtual mass force, Basselt force, gravity force and lift force are much smaller than the friction force.

Under these assumptions, the Euler equations governing time-dependent, two-dimensional and two-phase flows can be written in the quasi-conservative form as (in the following expressions, the subscript $p$ refers to the particles)

$$
\frac{\partial U}{\partial t}+\frac{\partial F(U)}{\partial x}+\frac{\partial G(U)}{\partial y}=H(U)
$$



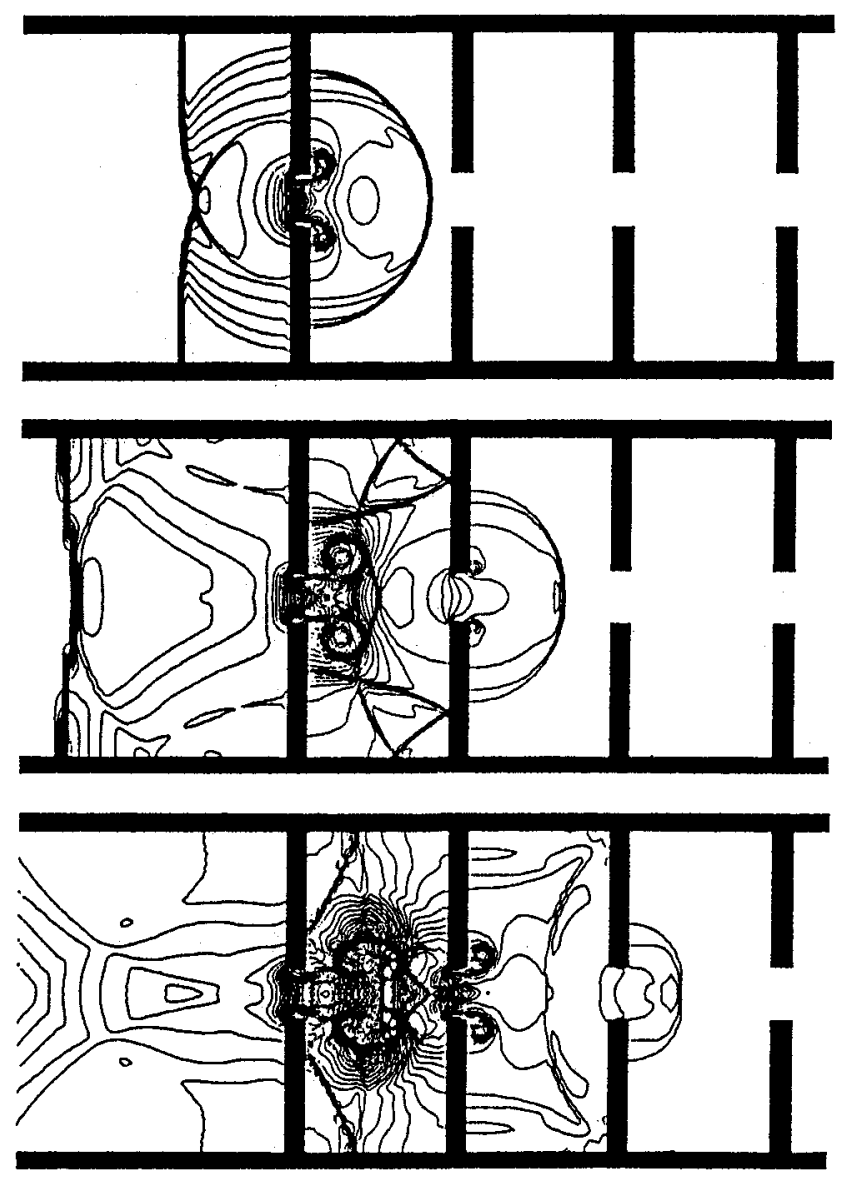

a
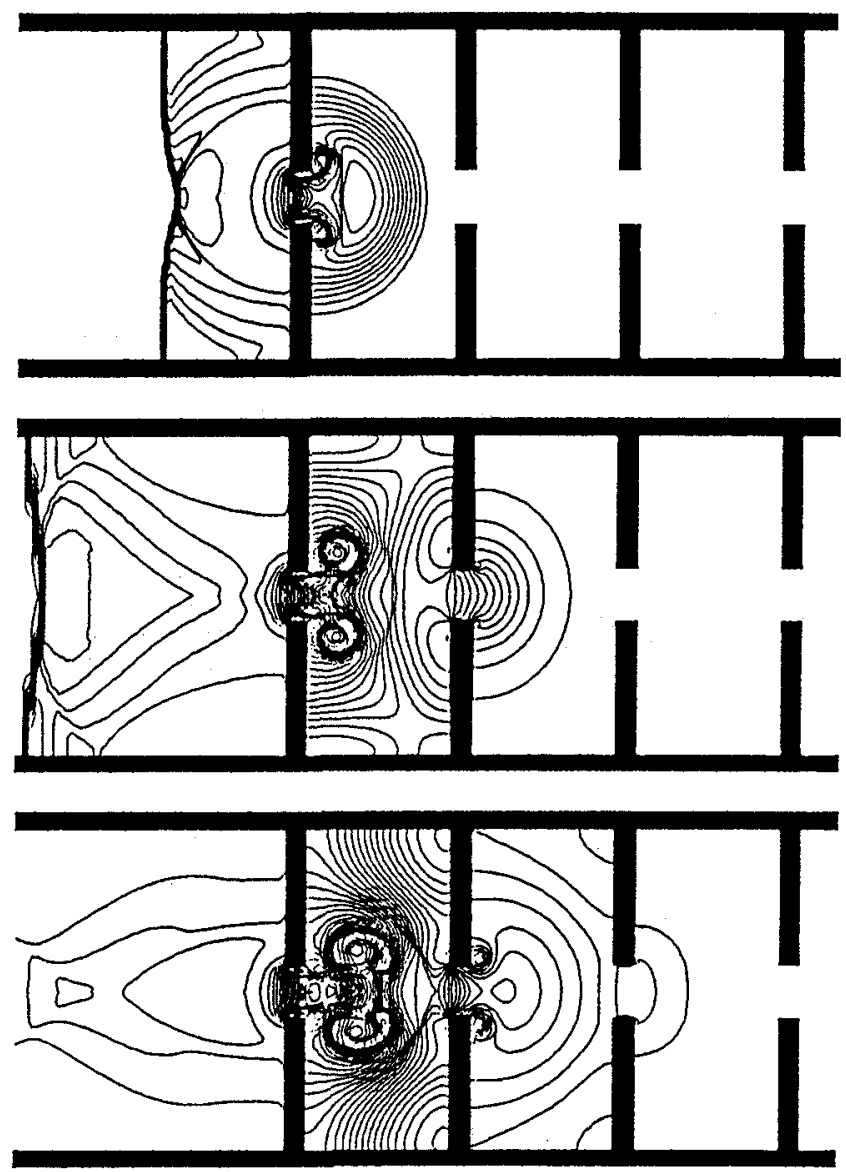

b

Fig. 1a,b. Density contours of gas phase $\left(M_{s}=1.23\right)$; a pure gas; $\mathbf{b}$ dusty gas

$$
U=\left(\begin{array}{l}
\rho \\
\rho u \\
\rho v \\
\rho E \\
\rho_{p} \\
\rho_{p} u_{p} \\
\rho_{p} v_{p} \\
\rho_{p} E_{p}
\end{array}\right), \quad F=\left(\begin{array}{l}
\rho u \\
\rho u^{2}+p \\
\rho u v \\
(\rho E+p) u \\
\rho_{p} u_{p} \\
\rho_{p} u_{p}^{2} \\
\rho_{p} u_{p} v_{p} \\
\rho_{p} E_{p} u_{p}
\end{array}\right)
$$$$
G=\left(\begin{array}{l}
\rho v \\
\rho u v \\
\rho v^{2}+p \\
(\rho E+p) v \\
\rho_{p} v_{p} \\
\rho_{p} u_{p} v_{p} \\
\rho_{p} v_{p}^{2} \\
\rho_{p} E_{p} v_{p}
\end{array}\right), \quad H=\left(\begin{array}{l}
0 \\
-f_{x} \\
-f_{y} \\
-Q \\
0 \\
f_{x} \\
f_{y} \\
Q
\end{array}\right)
$$

Here $u$ and $v$ are the velocity components in $x$ and $y$ directions. $E=C T+\left(u^{2}+v^{2}\right) / 2$ is the total specific energy ( $C$ stands for gas specific heat at constant volume $C_{v}$ or particle heat capacity $C_{m}$ ). The variables $p, \rho$, and $T$ are respectively pressure, density, and temperature. These three gas parameters can be associated by the state equation:

$$
p=\rho R T
$$

where $R$ is the gas constant. The appearance of some interphase terms is the main difference between our Equations
(1) for the dusty gas and the corresponding equations for the pure gas. For the present problem, the interaction parameters are introduced as follows: $f_{x}, f_{y}$, and $q$ are respectively the drag force components and heat transfer to be exterted on particles per unit volume; $Q=u_{p} f_{x}+v_{p} f_{y}+q$ is dissipation work due to the interphase effects.

\section{Numerical method}

In constructing a two-dimensional scheme for time-dependent and compressible flows, well-known techniques of operator-splitting for temporal and spatial operators (Sod 1978; Strang 1968) are employed and then the single 2-D conservation system (1) is decomposed into a set of three 1-D systems:

$$
\frac{\partial U}{\partial t}+\frac{\partial F(U)}{\partial x}=0 ; \frac{\partial U}{\partial t}+\frac{\partial G(U)}{\partial y}=0 ; \frac{d U}{d t}=H(U)
$$

In this way, there is no coupling between the flows of the gas and particle phases for the first two systems. As well known, when the shock wave propagates in the dusty gas, the induced flows for the gas and particle phases possess different features because that the solid particle has much larger inertia than the gas molecule. Hence, for solution of these first two equations, two different finite-difference schemes 

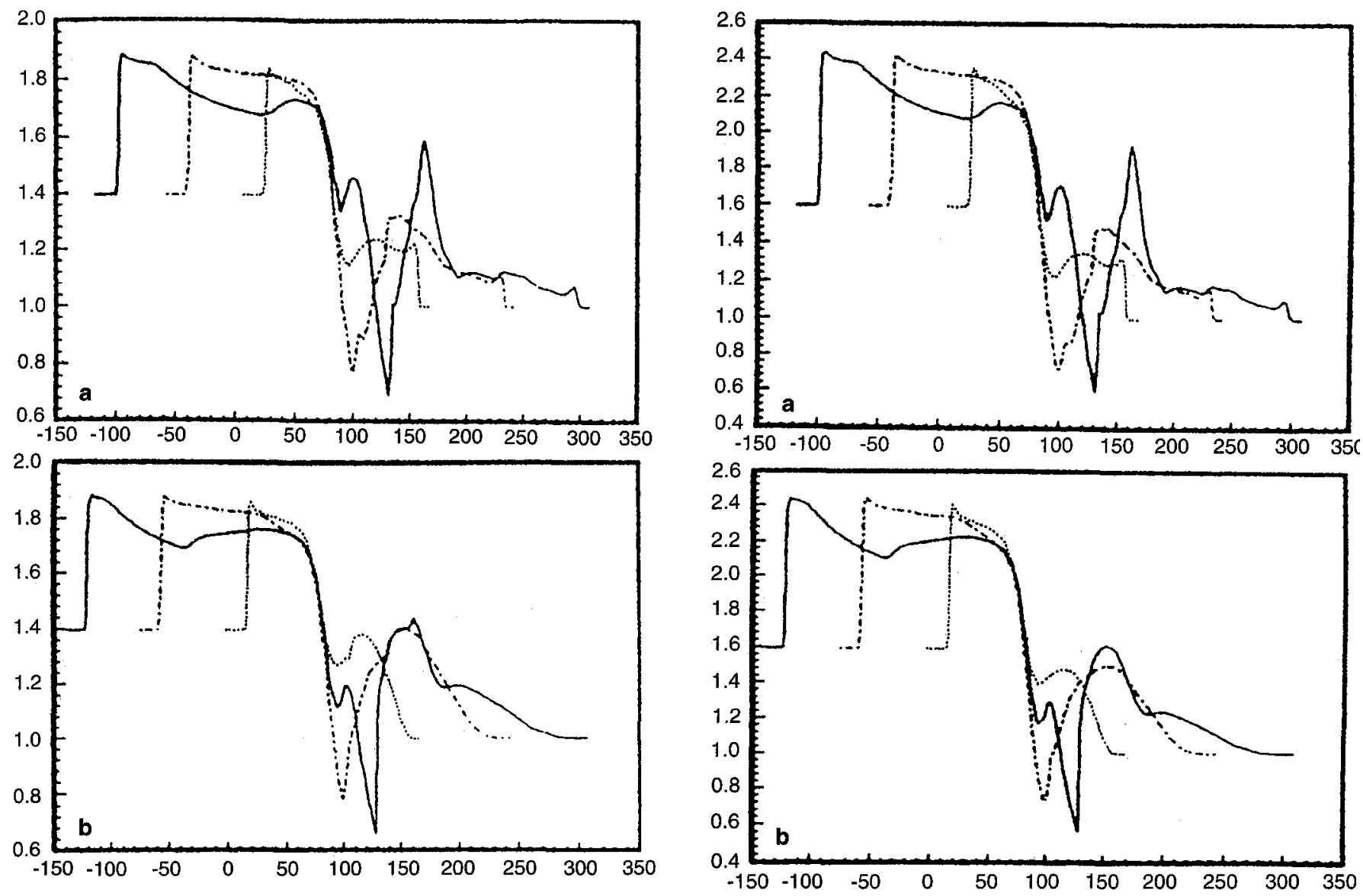

Fig. 2a,b. Density of gas phase along symmetric axis $\left(M_{s}=1.23\right)$; a pure gas; $\mathbf{b}$ dusty gas

based on the GRP and NND methods are applied respectively to solve the gas and particle flows. Here, the GRP method is an "analytic" second-order extension of the classical Godunov scheme (Godunov 1959). Due to its ability to capture shocks with high resolution without spurious oscillations, this upwind GRP scheme has been used for unsteady inviscid compressible flows of various fluids with jump discontinuities. Following the original work of Ben-Artzi and Falcovitz (1984), this method was applied to deal with 1D duct flows with material interfaces (Ben-Artzi and Birman 1986) and with variable cross section (Ben-Artzi and Falcovitz 1986), 2-D shock diffraction at a expansive corner (Hillier 1991), 2-D shock reflection by a double wedge (Falcovitz et al. 1993) and so on. Wang and her colleagues (1991, 1993) extended the GRP method to two-phase flows induced by shocks in a dusty gas. In the present paper, the operator-split GRP scheme is developed for predicting the complex shock-wave pattern involving transmission, reflection, diffraction and the resulting flow in the baffled tube. The NND method (Zhang 1988) is a non-oscillation and nonfree-parameter dissipation difference scheme and its NND-4 version (Zhang and Li 1992) has second-order accuracy. The third system is an ordinary differential equation which contains the interaction terms between the gas and particles. It is solved by a second-order predictor-corrector method.

Fig. 3a,b. Pressure of gas phase along symmetric axis $\left(M_{s}=1.23\right)$; a pure gas; $\mathbf{b}$ dusty gas

Let $L x, L y$, and $L r$ to be the difference operators. The following combined five-step scheme can be constructed for each time step:

$$
\begin{aligned}
U_{i j}^{n+1} & =\operatorname{Lr}\left(\frac{\Delta t_{n}}{2}\right) \operatorname{Lx}\left(\frac{\Delta t_{n}}{2}\right) \\
& \times \operatorname{Ly}\left(\Delta t_{n}\right) \operatorname{Lx}\left(\frac{\Delta t_{n}}{2}\right) \operatorname{Lr}\left(\frac{\Delta t_{n}}{2}\right) U_{i j}^{n}
\end{aligned}
$$

where $i, j$ and $n$ are space and time nodes; $\Delta t_{n}$ is the time interval determined by the CFL condition to satisfy the stability requirement.

\section{Computational results}

With the above algorithm, computations were performed to simulate the dusty shock reflection and diffraction process in the aligned 4-baffle system with the following parameters for the particle phase: diameter $d=10 \mu \mathrm{m}$, material density $\sigma_{p}=$ $2500 \mathrm{~kg} / \mathrm{m}^{3}$, mass loading ratio $\alpha=1.0$, heat capacity ratio $\beta=1.0$ and with the following expressions for the interphase interactions: drag coefficient $C_{D}=0.48+28 R e^{-0.85}$, Nusselt number $N u=2.0+0.6 \operatorname{Pr}^{1 / 3} \operatorname{Re}^{1 / 2}$ (Re is the slip Reynolds number, $\mathrm{Pr}$ is the Prandtl number). Corresponding calculations for the pure gas were also carried out in order 

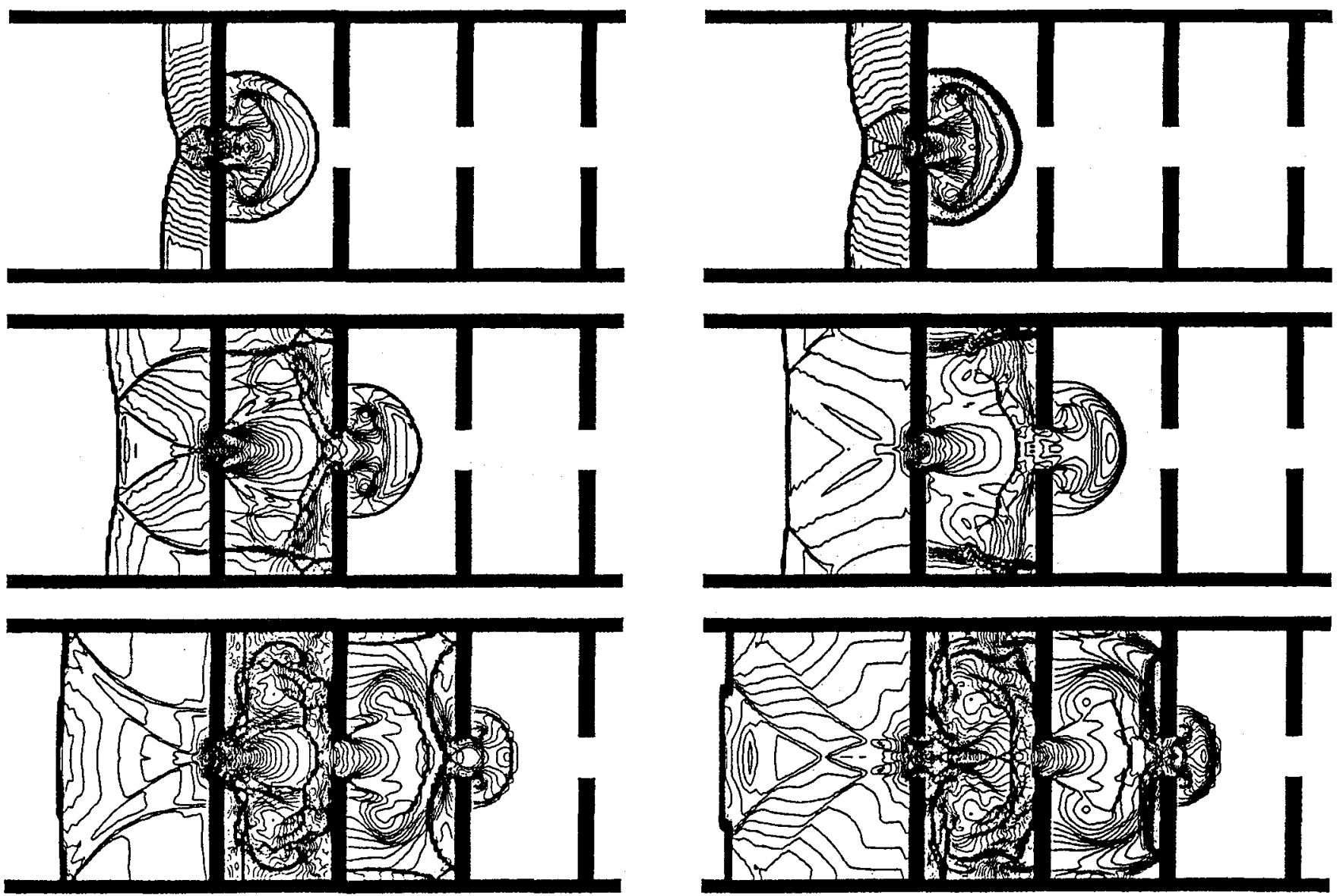

Fig. 4a,b. Density contours of gas phase $\left(M_{3}=2.50\right)$; a pure gas; $\mathbf{b}$ dusty gas
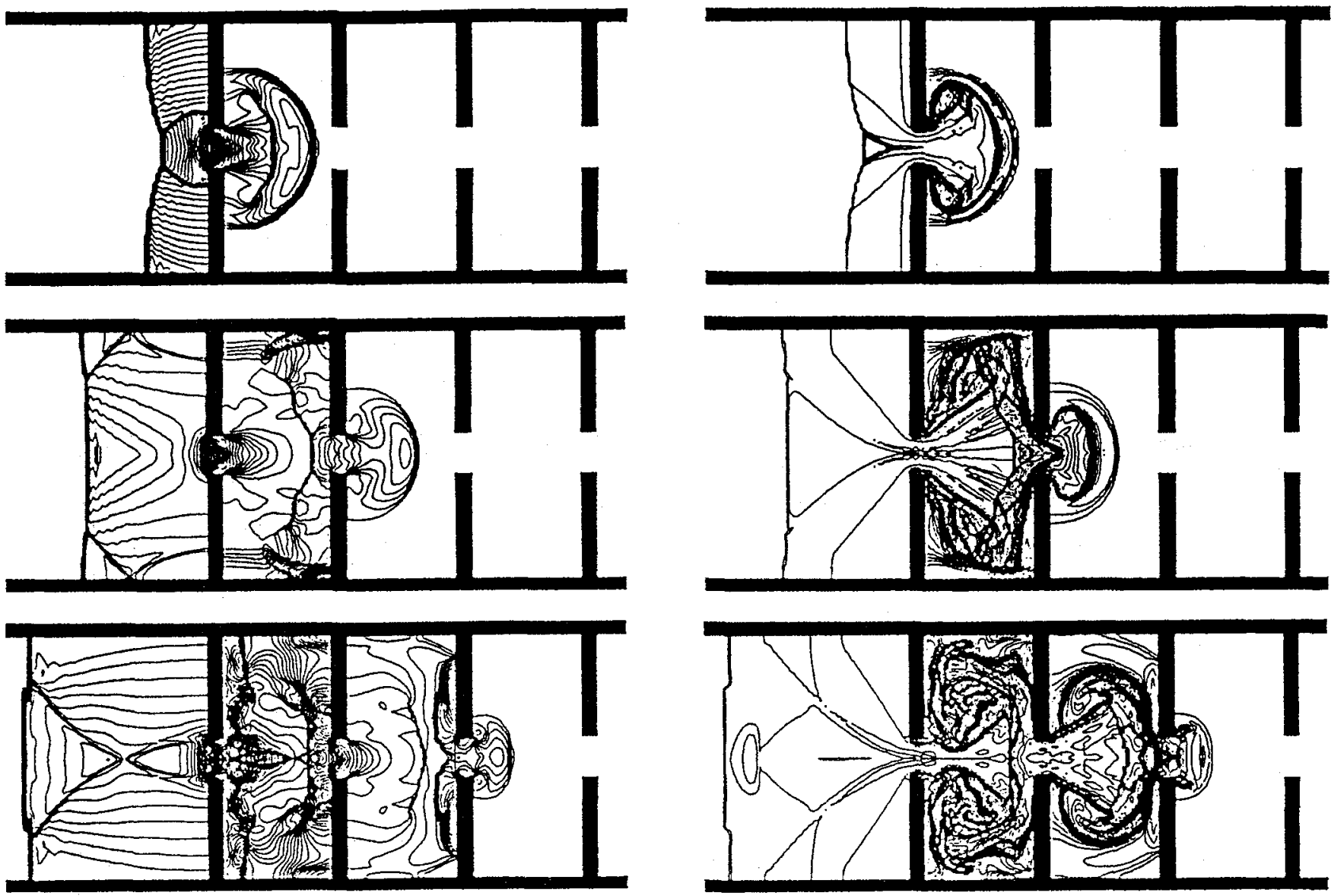

Fig. 5a,b. Shock-induced gas flow field in dusty gas $\left(M_{s}=2.50\right)$; a pressure contours; $\mathbf{b}$ entropy contours 


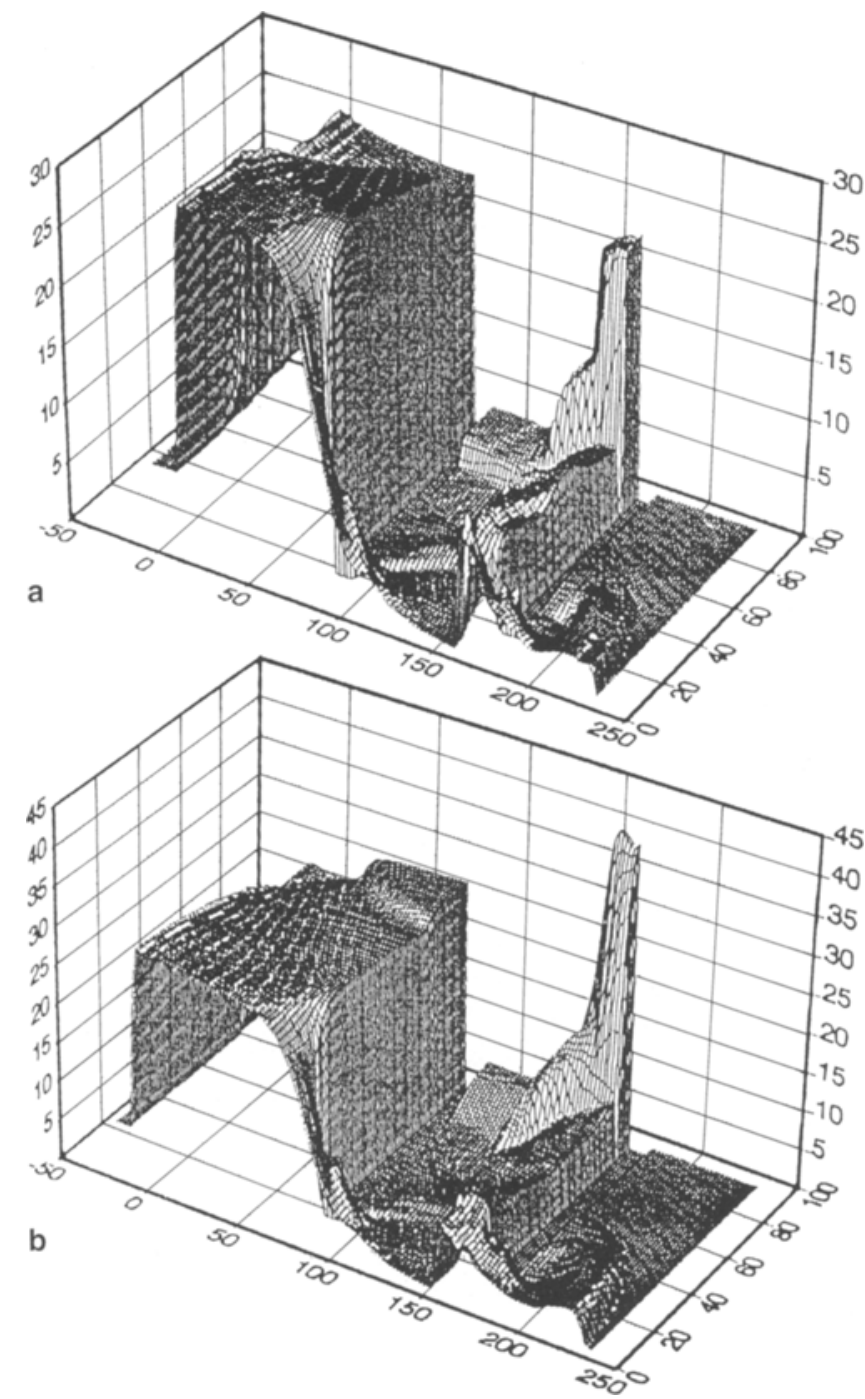

Fig. 6a,b. Pressure distributions of gas phase in baffle system $\left(M_{s}=2.50\right)$; a pure gas; $\mathbf{b}$ dusty gas

to illustrate particle effects on the induced flows. The computation was first made under the same conditions as for the pure gas interferogram test by Reichenbach and Kuhl (1993), where the incident shock Mach number $M_{S}$ is 1.23. In this weak-shock case, the obtained isopycnics for the pure and dusty gases are plotted in Fig. $1 \mathrm{a}$ and $1 \mathrm{~b}$, respectively. The time sequence of the flow field in Fig. 1a takes the same instants as the flow visualization test: they are successively at $t=96.04 \mu \mathrm{s}, 186.08 \mu \mathrm{s}$ and $282.35 \mu \mathrm{s}$ (here, the moment at which the shock front arrives the first baffle plate is taken as the initial time $t=0$ ). The comparison of numerical and experimental results indicates the excellent agreement between the computation and experiment. It verifies the accuracy and effectiveness of the numerical method. Figure $1 \mathrm{~b}$ presents the numerical results of the shock-induced flow in the dusty-gas case, where the three frozen shock fronts have the same $x$-position as for the pure gas. However, the arrival time takes different values as $t=108.94 \mu \mathrm{s}, 220.61 \mu \mathrm{s}$ and $315.65 \mu \mathrm{s}$. The decrease in the strength and speed of the transmitted shock front can be attributed to particle dissipation mechanisms. The variations of gas density along

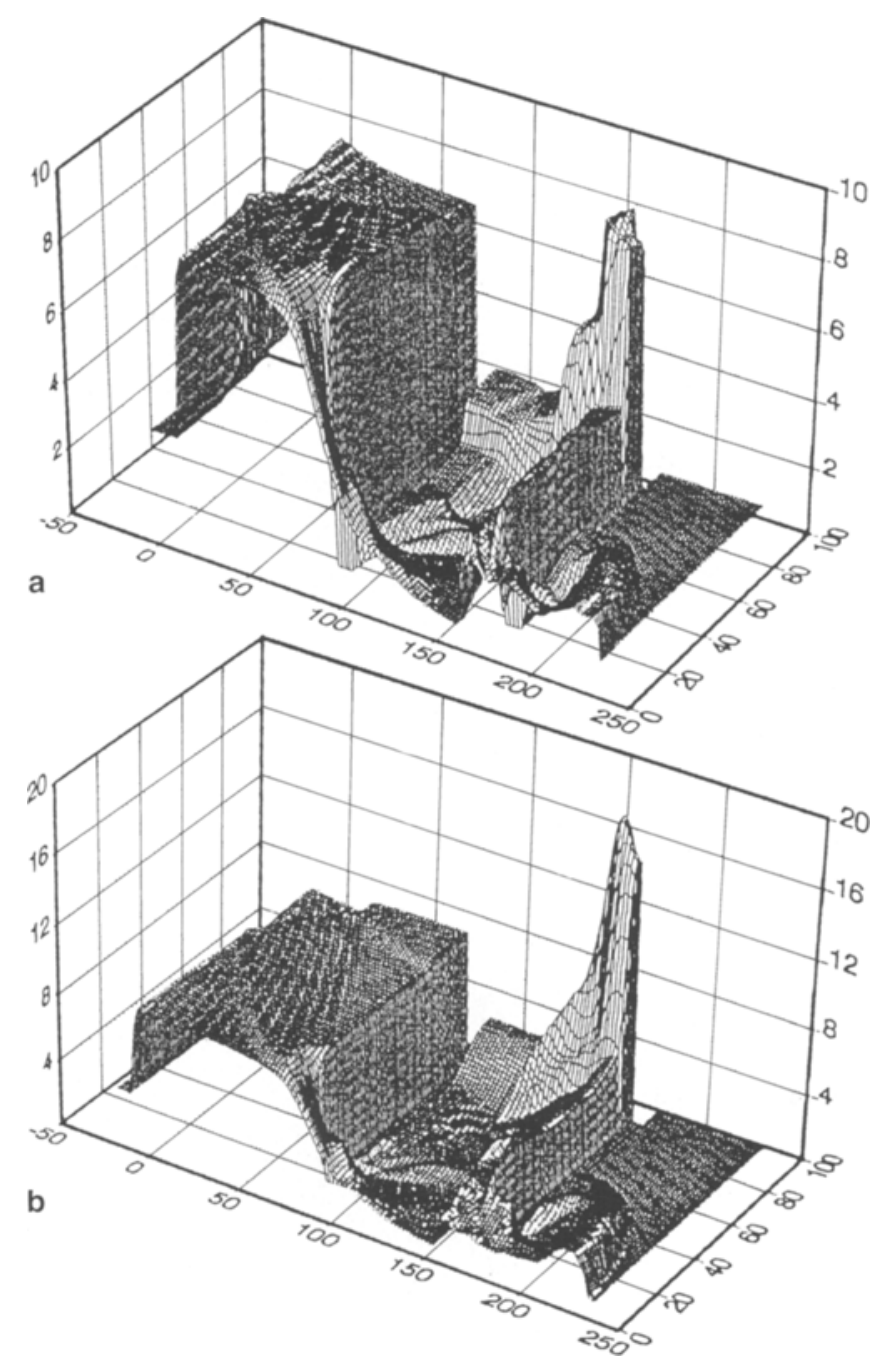

Fig. 7a,b. Density distributions of gas phase in baffle system $\left(M_{s}=2.50\right)$; a pure gas; $\mathbf{b}$ dusty gas

the symmetric axis are shown in Fig. 2a (for the pure gas) and $2 b$ (for the dusty gas). Similarly, the variations of gas pressure along the symmetric axis are given in Fig. 3a (for the pure gas) and $3 \mathrm{~b}$ (for the dusty gas). In Figs. 2 and 3 the position $x=0$ is taken at the first baffle plate. It is seen that under the weak-shock condition, the shock front is fully dispersed in the dusty-gas case. For a stronger incident shock wave $\left(M_{S}=2.50\right)$, Fig. 4 shows the gas density contours in the post-shock flow field. The time instants in sequence are respectively $t=48.65 \mu \mathrm{s}, 104.71 \mu \mathrm{s}, 160.11 \mu \mathrm{s}$ for the pure-gas case and $t=61.43 \mu \mathrm{s}, 132.21 \mu \mathrm{s}, 198.69 \mu \mathrm{s}$ for the dusty-gas case. In addition, for the dusty-gas case, the pressure and entropy contours of the gas phase at the three different time are given respectively in Fig. 5a and 5b. Similarly, at these moments the shock front arrives at the same position as in the $M_{S}=1.23$ case. In Figs. 6 and 7 the pressure and density distributions of the gas phase at the time $t=132.21 \mu \mathrm{s}$ are presented for the case of $M_{s}=2.50$. Compared with the pure-gas case, the overpressure in the dusty-gas case is much higher due to the presence of particles in the baffle system (cf. Figs. 6a and 6b). This fact can be found from Fig. 8 where the density distributions of 


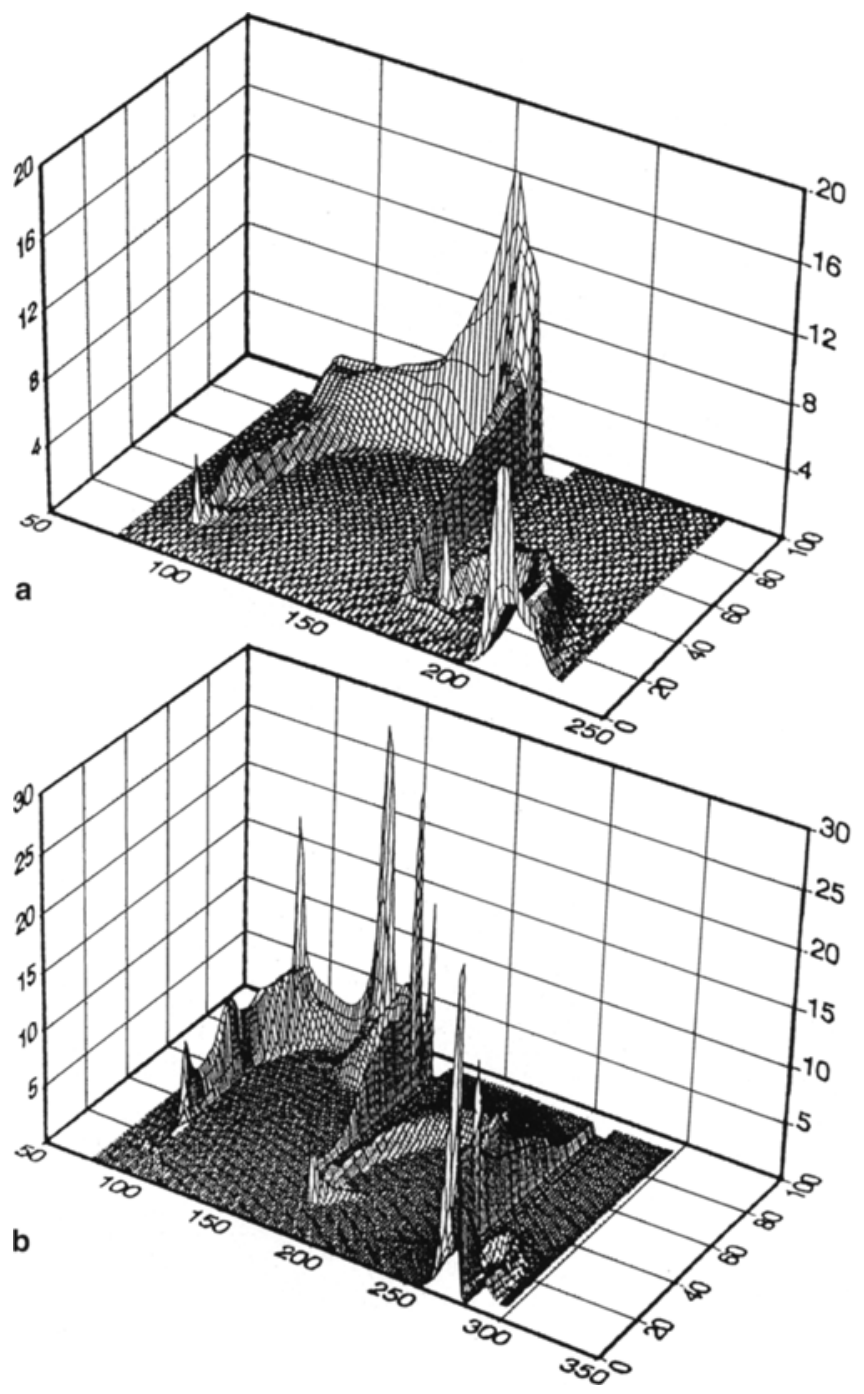

Fig. 8a,b. Density distributions of particle phase in baffle system $\left(M_{s}=\right.$ $2.50) ; \mathbf{a} t=132.21 \mu \mathrm{s} ; \mathbf{b} t=198.69 \mu \mathrm{s}$

the particle phase at the time $t=132.21 \mu \mathrm{s}$ and $198.69 \mu \mathrm{s}$ are plotted. These numerical results reveal the fundamental features of the dusty shock reflection and diffraction patterns. When an incident shock wave propagates through a dusty gas in a baffled tube, reflection and diffraction occur and the strength of the transmitted shock is greatly reduced because of double mechanisms: baffle blocking and particle dissipation. For the dusty gases, the reflection and diffraction patterns in baffle systems become more complicated compared with those in corner systems(Wang et al. 1993). It is seen that, at each sharp corner of the orifices in the baffule plate, a shear layer is generated and then rapidly rolls up in a spiral vortex. Moreover, each pair of the corners results in a mushroom-shaped region, where turbulent mixing is a dominant process. Therefore turbulent mixing, particle relaxation, and their interactions consist of complex fluid-dynamic effects.

\section{Conclusion}

Numerical simulations of the dusty-shock-induced flow in the aligned two-dimensional baffle system indicate that the reflection and diffraction patterns become quite complex due to the presence of baffle plates and particles. Turbulent mixing and particle relaxation are the main dissipation mechanisms.

Acknowledgement. The authors are indebted to the National Natural Science Foundation of China for its financial support.

\section{References}

1. Ben-Artzi M and Birman A (1986) Application of the "Generalized Riemann Problem" Method to 1-D Compressible Flows with Material Interfaces. J. Comp. Phys. 65:170-178

2. Ben-Artzi M and Falcovitz J (1984) A Second-order Godunov-type Scheme for Compressible Fluid Dynamics. J. Comp. Phys. 55:1-32

3. Ben-Artzi M and Falcovitz J (1986) An Upwind Second-order Scheme for Compressible Duct Flows. SIAM J. Sci. Stat. Comp. 7:744-768

4. Falcovitz J, Alfandary G and Ben-Dor G (1993) Numerical Simulation of the Head-on Reflection of a Regular Reflection. Int. J. Numerical Method in Fluids 17:1055-1077

5. Godunov SK (1959) A Finite Difference Method for the Numerical Computation of Discontinous Solutions of the Equations of Fluid Dynamics. Mat. Sbornik 47:271-295

6. Hillier R (1991) Computation of Shock Wave Diffraction at a Ninety Degrees Convex Edge. Shock Waves 1:89-98

7. Hillier R (1993) Numerical Modelling of Shock Wave Diffraction. In: Proc. of 19th Int. Symp. on Shock Waves (Ed. R. Brun), SpringerVerlag, Vol.4, pp 17-26

8. Luo H, Baum JD, Lohner R and Cabello J (1993) Adaptive Edge-based Finite Element Schemes for the Euler and Navier-Stokes Equations on Unstructured Grids. AIAA-93-0336.

9. Reichenbach H and Kuhl AL (1993) Shock-Induced Turbulent Flow in Baffle Systems. FhG/EMI E12/93

10. Sod GA (1978) A Survey of Several Finite Difference Methods for Systems of Nonlinear Hyperbolic Conservation Laws. J. Comp. Phys. 27:1-31

11. Strang $G$ (1968) On the Construction and Comparison of Difference Schemes. SIAM J. Num. Anal. 5:506-517

12. Wang BY and Wu SQ (1991) Numerical Investigation of Dusty Gas Shock Wave Propagation along a Variable Cross-section Channel. In: Proc. of 18th Int. Symp. on Shock Waves (Ed. K. Takayama), SpringerVerlag, pp 521-526

13. Wang BY, Wu SQ, Zhu H and Xu YH (1993) Numerical Simulation of Dusty Shock Diffraction Around a 90 Degree Corner. In: Proc. of 19th Int. Symp. on Shock Waves (Ed. R. Brun), Springer-Verlag, Vol.3, pp 61-66

14. Zhang HX (1988) Non-oscillation and Non-free-parameter Dissipation Difference Scheme. Acta Aerodynamica Sinica 6:143-165

15. Zhang HX and Li ZW (1992) Numerical Simulation of Hypersonic Laminar Wake Flow. Acta Mechanica Sinica 24:389-399

This article was processed by the author using the $\mathrm{IAT}_{\mathrm{E}} \mathrm{X}$ style file pljour 2 from Springer-Verlag. 